\title{
Recent Understanding of Peritoneal Pathology in Peritoneal Dialysis Patients in Japan
}

\author{
Chieko Hamada $^{a}$ Yasuhiko Tomino ${ }^{b}$ \\ ${ }^{a}$ Advanced Research Institute for Health Science, Faculty of Medicine, Juntendo University, Tokyo, Japan; \\ ${ }^{b}$ Asian Pacific Renal Research Promotion Office, Medical Corporation SHOWAKAI, Tokyo, Japan
}

\section{Keywords}

Peritoneal sclerosis · Encapsulating peritoneal sclerosis ·

Neutral peritoneal dialysis fluid · Pathological finding ·

Peritoneal dialysis

\section{Abstract}

The thin peritoneum covering the peritoneal cavity has been used as a dialysis membrane for peritoneal dialysis (PD) because it is highly vascularized and has a large body surface area. However, it has been reported that peritoneal membranes affected by peritonitis, as well as those exposed to the nonphysiological high glucose levels containing PD dialysate, may undergo histological and functional changes. Patients undergoing PD may experience encapsulating peritoneal sclerosis (EPS), which is a life-threatening serious complication of PD that can significantly impair activities of daily living. The incidence of EPS was $1.4-7.3 \%$ of maintenance PD patients in the 1980s. The incidence has improved to $1.0 \%$ after a neutral dialysate became the standard PD treatment in Japan. Furthermore, the pathogenesis of EPS is uncertain although its onset may be explained by the "twohit theory," in which some factors leading to impairment had an additive effect on simple peritoneal sclerosis. The evalua-

karger@karger.com www.karger.com/bpu

Karger $\stackrel{\text { ' }}{=}$
(C) 2021 The Author(s)

Published by S. Karger AG, Basel

This article is licensed under the Creative Commons AttributionNonCommercial-NoDerivatives 4.0 International License (CC BYNC-ND) (http://www.karger.com/Services/OpenAccessLicense) Usage and distribution for commercial purposes as well as any distribution of modified material requires written permission. tion of histopathological findings has shown the impact of the neutral dialysate on peritoneal deterioration as well as its role in the development of functional changes. In the present report, we discuss the advances in the understanding of peritoneal deterioration based on histological and macroscopic evaluations of the peritoneum of patients undergoing PD. We also discuss the recent treatment for PD patients.

(C) 2021 The Author(s)

Published by S. Karger AG, Basel

\section{Basic Structure and Function of the Peritoneum}

The first report on peritoneal tissue, published in 1930 by James Douglaus, explained that "peritoneum is a smooth and extremely thin membranous tissue that plays a role in the peristaltic motion of the gastrointestinal tract and wraps the abdominal organs." Douglaus also described the peritoneum as "a tissue covered with flat cells in a single layer, and within this thin membrane, there is a binding weave, of cells, vessels, lymph vessels, nerve fibers, and other structures, with a thickness of 30-40 $\mu \mathrm{m}$."

The peritoneum consists of the parietal peritoneum covering the abdominal wall and pelvic cavity, and vis- 
Fig. 1. Histological findings of the peritoneum. a The findings of the peritoneum at the initiation of PD (PAS. $\times 100)$. $\mathbf{b}$ The alteration in the peritoneum of a patients undergoing PD. Interstitial thickening is evaluated by the average of the submesothelial compact zone thickness at 5 points $(\mathrm{EV} . \times 40)$. Figure in the lower left shows post-capillary venule. Vasculopathy is evaluated by vascular patency in post-capillary venules and blood vessel density per area (PAS. $\times 200$ ). $\mathrm{PD}$, peritoneal dialysis; EV, Elastica van Gieson; PAS, periodic acid-Schiff.

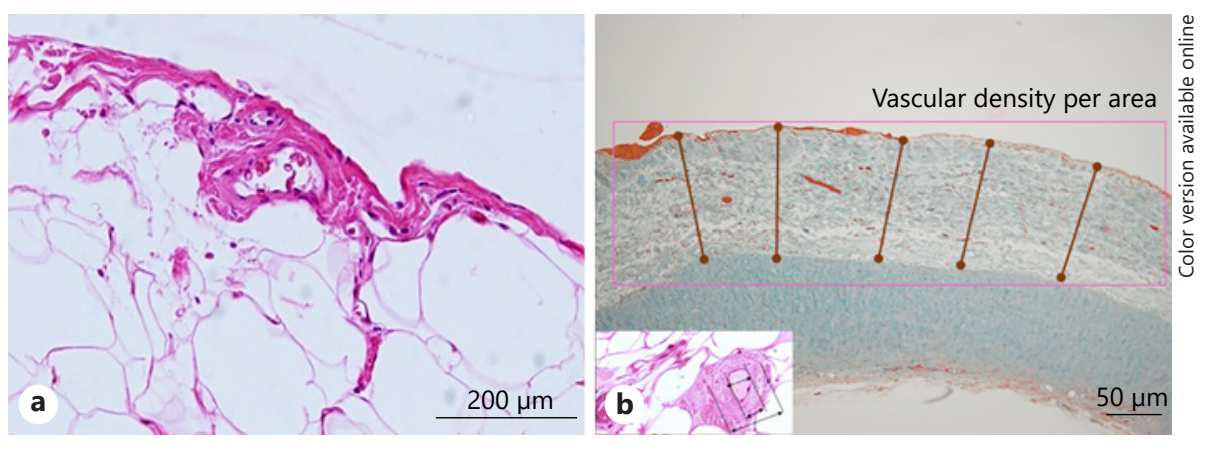

ceral peritoneum covering the abdominal organs (mainly the intestinal tract), mesentery, and omentum. The basic peritoneal construction is a mesothelial cell monolayer covering the peritoneal surface and the connective tissue just beneath it [1]. The high elasticity and plasticity of the peritoneum are a result of that action of the mesothelial cells covering the peritoneal surface. These cells have fine microvilli $(3-12 \mu \mathrm{m})$ on their surface and synthesize and secrete various lubricating substances such as hyaluronic acid [2]. The connective tissue under the basement membrane of the mesothelial cells has various mesenchymal components, such as mesoderm-derived blood vessels, lymphatic vessels, adipose tissue, and collagenous fibers, and the parietal peritoneum is continued to the fascia and muscle in the abdominal wall (Fig. 1a). On the peritoneum covering the omentum, a lymphoid tissue, that is, milky spots, and a pathway that allows lymphocytes and macrophages to enter the abdominal cavity freely through small defective holes called stomata (about $5 \mu \mathrm{m}$ in size) can be found $[1,3]$.

\section{Peritoneal Deterioration by Conventional Acidic High Glucose Degradation Product Dialysate}

\section{Histology of Hyalinizing Peritoneal Sclerosis (Simple Peritoneal Sclerosis)}

Peritoneal deterioration involves both functional failure and morphological alteration of the peritoneum when it is used as a dialysis membrane. Functional failure is characterized by ultrafiltration failure and peritoneal permeability enhancement. Morphological alterations of the peritoneum are judged based on macroscopic and microscopic findings, and mesothelial cytology of the peritoneal effluent [4-6]. The evaluation of the macroscopic findings of the peritoneum in a patient using an acidic dialysate is characterized by morphological alterations such as changes in the color of the peritoneum (i.e., it appears brown or tanned in color) and changes in its texture (i.e., there is a loss of surface moisture, which grants it a leather-like appearance) [4]. Histologically, morphological alterations are characterized by the disappearance of mesothelial cells, fibrosis and thickening of the mesothelial interstitium, thickening of the walls and stenosis/occlusion of the lumen of small blood vessels, angiogenesis, and lymphatic vessel proliferation [5, 6] (Fig. 1b). Williams et al. [7] in 2002 and Honda et al. [8] in 2008 proposed the quantification (semi-quantification) method of the histological characteristics of the peritoneum of patients undergoing peritoneal dialysis (PD). They proposed that uremic condition and PD duration play important roles in the development of peritoneal deterioration.

\section{Factors Related to Peritoneal Deterioration}

Underlying conditions that lead to uremia, such as diabetes, uremic toxins, drugs, peritonitis, and various kinds of biostimulatory factors, deterioration of $\mathrm{PD}$ equipment and so on are the causes of peritoneal deterioration [9]. In particular, peritonitis and biocompatibility of the peritoneal dialysate may play crucial roles in peritoneal injury. The acidity, high concentration of lactic acid, high osmotic pressure, and high glucose concentration in the peritoneal dialysate, and glucose degradation product (GDP) can be cited as the factors contributing to peritoneal deterioration $[10,11]$. In general, as the peritoneal disorders are enhanced by the prolongation of the $\mathrm{PD}$ period, peritoneal injury induced by the PD dialysate is thought to be a major cause of peritoneal deterioration. According to the reports on the effect of reducing peritoneal injury due to low GDP dialysate in PD patients described below, GDP is a major responsible factor for peritoneal deterioration among several bio-incompatible factors in conventional acidic PD solution. 
Relationship between Morphological and Functional Changes in Peritoneal Deterioration

The relationship among ultrafiltration failure, peritoneal hyperpermeability, and morphological changes in peritoneal pathology remains unclear. Rippe [12] proposed the mechanism of the vascular endothelial layer, which is the fundamental concept of material permeability in the peritoneum, that is, the three-pore model. It is assumed that pores of 3 sizes in the vascular endothelial layer facilitate solute transport; these are ultra-small, small, and large pores. The ultra-small pore allows water molecules to pass, and it is composed of aquaporin-1. The pores for uremic nitrogen and Cr transport are the small pores, which correspond to the cleft and gap between vascular endothelial cells in capillaries. It is thought that the intercellular gap of the venule plays the role of the large pore, which facilitates the transport of medium and large molecules, such as albumin. The regulation of the gap junction is associated with the cellular thickness and the condition of the endothelial cells. Aside from endothelial intercellular transfer, medium and large molecules are also transported via intracellular transport (transcytosis). It is postulated that caveolin-1, a major constituent protein of caveolae on the surface of endothelial cells, is involved in the transport of medium and large molecules by regulating endothelial cell integrity. Recent studies have suggested that the glycocalyx, which is a mucopolysaccharide covering the surface of the vascular endothelial layer and produced by vascular endothelial cells, is important for vascular permeability [13]. Glycocalyx in the endothelial surface is thought to function as a first barrier for the transport of medium and large molecules in the vascular endothelium $[14,15]$.

In healthy people, the peritoneal microcirculation increases in density once in the fetal period but remains constant throughout life. However, angiogenesis is induced by peritonitis, cancer metastasis, and invasion such as surgery [3]. Ultrafiltration failure is a symptom of peritoneal hyperpermeability, in which the osmotic gradient between the plasma and dialysate cannot be maintained, as glucose, an osmotic substance in the dialysate, rapidly moves into the blood vessel from the peritoneal cavity. Glucose, as well as other small molecules such as $\mathrm{Cr}$ and urea, is rapidly transferred from the peritoneal cavity to peritoneal blood vessels because of the state of hyperpermeability [16-18]. Diffusion of small molecule substances has been shown to be caused by an increase in the number of blood vessels induced by vascular endothelial growth factors and inflammatory cytokines, as well as an increase in an area of the vascular bed due to vasodilation and loosening in the vascular endothelial cell junction [7, 19]. Gavard and Gutkind [20] pointed out VEGF, which regulates intracellular adhesion molecule such as VE cadherin, is one of the most potent inducer increasing vascular permeability on endothelial cells. Flessner et al. [21] showed that alteration of the intestinal matrix, such as peritoneal thickening and increase of hyaluronan, correlated with diminished hydraulic conductivity and macromolecular transport in an animal model in which a highglucose concentration solution is repeatedly injected into the abdominal cavity. Intraperitoneal administration of conventional peritoneal dialysate for 6 weeks induced angiogenesis, and a correlation was observed between blood vessel density and hyaluronan concentration in PD fluid [22]. Osada et al. [23] suggested that stroma, as well as perivascular proteoglycan composition, was different at the beginning of PD and at the end of long-term PD. Degeneration of interstitial collagen and specific vascular lesions are strongly affected by advanced glycation end product (AGE) formation in extracellular matrix components. It is thought that the cross-linking change of collagen molecules by AGE formation causes the structural alteration of the barrier proteins and leads to hyperpermeability. In addition, it is thought that the permeability of small molecules, as well as medium and large molecules, is enhanced by an impaired barrier function of the endothelial cells induced by vascular endothelial injury due to GDP and AGE.

\section{Peritoneal Sclerosis - Simple Peritoneal Sclerosis and Sclerosing Peritonitis (Fig. 2)}

\section{Historical Review}

Peritoneal sclerosis is a comprehensive concept, including not only fibrosis of the interstitium but also the degeneration of fibrotic components, such as collagenous fibers in the peritoneal interstitium and vascular walls $[2$, $4,24]$. Peritoneal disorders can be pathologically designated into 2 categories: simple peritoneal sclerosis and encapsulating peritoneal sclerosis (EPS), formerly called sclerosing peritonitis. Until now in Japan, peritoneal injury has been described using terms such as peritoneal fibrosis, peritoneal sclerosis, sclerosing encapsulating peritonitis, and EPS [25]. Recently, as a term corresponding to overseas peritoneal fibrosis (Fig. 2a), peritoneal sclerosis is similarly divided into simple peritoneal sclerosis (regarded as hyalinizing peritoneal sclerosis [HPS]) (Fig. 2a) and EPS (Fig. 2b). In the case of HPS, the parietal peritoneum and the visceral peritoneum have shown uni- 
Fig. 2. Histological findings of peritoneal sclerosis. a Simple peritoneal sclerosis (HPS) (PAS. $\times 100)$. b Peritoneal sclerosis covered with deposited fibrin (arrow heads). The stroma is markedly thickened by the accumulation of degenerated collagen fibers, and the small vascular vessels in the interstitium are obstructed. Infiltration of inflammatory cells is also observed in the interstitium (arrows) $($ HE. $\times 100)$. HPS, hyalinizing peritoneal sclerosis; HE, hematoxylin eosin.
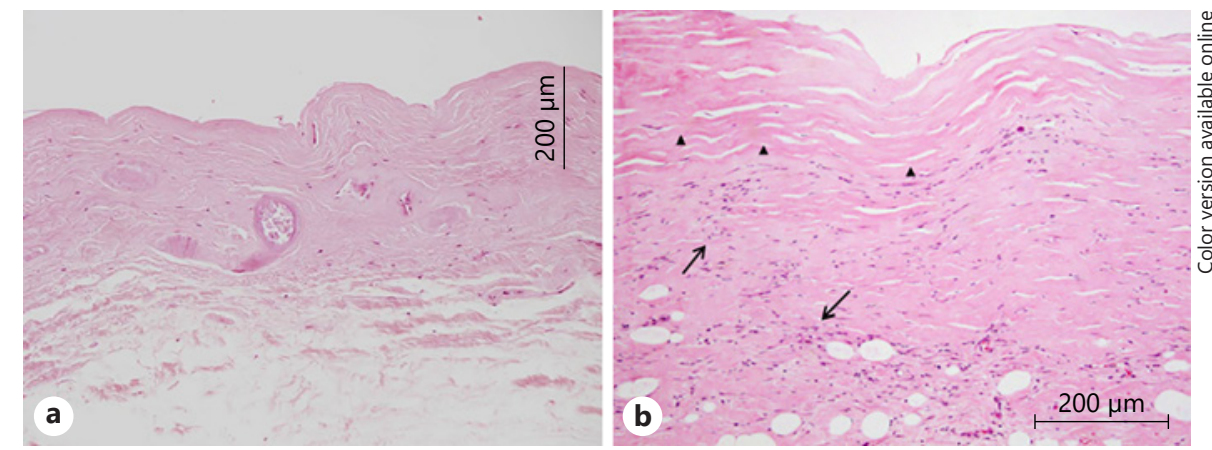

form fibrous thickening of the submesothelial interstitium ranging from 200 to $500 \mu \mathrm{m}$ and sometimes up to 1 $\mathrm{mm}$, along with the degeneration of the collagenous fibers and vascular wall sclerosis [26, 27] (Fig. 1b, in inset). In 1998, the Ministry of Health and Welfare, Ministry of Chronic Disease Research Project, and the Chronic Renal Failure Research Group (Research Group on Evaluation and Appropriateness of CAPD Therapy) in Japan defined EPS as a "sustained, intermittent or repetitive condition depending on the extension of the adhesion and diffuse thickening of the peritoneum. It is a syndrome that exhibits symptoms of ileus and therefore is a clinical entity."

The first report of EPS goes back to 1980 [28]. The incidence of EPS was 1.4-7.3\% of maintenance PD patients in the 1980s. However, in the report of Iwatsuki et al. [29] in 1998 , it was $1.7 \%$, a slightly lower rate than that found in Europe and the United States. According to a report by Kawanishi et al. [30], the incidence rate was 2.5\% in 2004. In 2014, Nakayama et al. [31] reported an improved tendency of $1.0 \%$ after a neutral dialysate became the standard PD treatment in Japan. Previously, EPS was a seriously severe complication of PD that caused mortality in $25.8-56.5 \%$ of the affected patients [30, 32, 33]. The dramatic reduction of EPS patients and the decline of EPSrelated death reported in the NEXT-PD study in 2014 [30] show that the prevention and treatment strategies for EPS in Japan have been effective in recent years. As the risks of EPS development, (a) long-term continuation of PD, (b) enhancement of peritoneal permeability, and (c) peritonitis are mentioned. Whether simple peritoneal sclerosis, which recognizes fibrous hyperplasia of the peritoneal stroma and vasculopathy in microvessels, is associated with the onset of EPS remains controversial [17, 34]. In the era of acidic peritoneal dialysate, simple peritoneal sclerosis or HPS were considered as a strong predisposing factor for development of EPS; however, the recent epidemiological studies have been clarified that it does not always predispose patients with EPS. EPS pa- tients have 3.3 times more frequent history of peritonitis compared with non-EPS patients [35]. In another study, $5.9-17.2 \%$ of the PD patients who had been treated with an acidic dialysate over 8 years had developed EPS [36].

\section{Pathological Findings and Pathogenesis}

Because the inflammatory findings are not a central feature of this disease, the name was changed from sclerosing encapsulated peritonitis to EPS in the report of the International Society of Peritoneal Dialysis (ISPD) Ad Hoc Committee in 2000 [35]. The characteristic morphological finding of EPS is the formation of a fibrous encapsulating membrane that envelops the intestines [37]. Sherif et al. [38] reported that fibrin exudation on the peritoneal surface was often found in patients with EPS as compared with the non-EPS patients, and the thickness of the degenerated collagen layer increased. A macroscopic feature found in patients with EPS is a white film-like structure, consistent with a newly formed membrane (NFM) on the surface of the visceral peritoneum [39]. Histologically, the film-like structure is composed of exudated fibrin deposits, blood, infiltrating inflammatory cells, and proliferating fibroblasts. Honda et al. [40] proposed a "two-hit theory" to explain the development of EPS, in which the peritoneum is deteriorated by exposure to the bio-incompatible dialysate (first hit) and then is exposed to additional deteriorating factors (second hit). However, EPS is often developed without any trigger (second hit). Peritoneal deterioration (simple peritoneal sclerosis) is an important background factor for the development of EPS. As described in the previous chapter, excessive increase of peritoneal permeability by a vascular endothelial injury caused by GDP or AGE promotes exudation of fibrin. Subsequently, the layer of fibrin membrane covers the intestines under an imbalanced coagulation/fibrinolytic system in the peritoneal cavity [39]. As time passes, the exudated fibrin membrane is organized, and the component develops into firm envelopes mainly composed of fibrous collagen [41]. 
Clinical and Pathological Staging of EPS

I believe that the presentation of clinical staging and treatment strategies based on clinical and imaging findings and histological findings contributes significantly to EPS treatment strategies in Japan (Table 1). Table 1, which is based on references [35] and [37]. Honda et al. [39] classified 8 cases diagnosed with EPS by enterolysis surgery into 3 histological stages according to the phenotype of the NFM by the presence of precipitated fibrinand podoplanin-positive fibroblasts in the dissected peritoneal specimens. Then, they examined the relationship between histological and clinical findings [39]. In the exudative phase (stage I), fibrin deposition is apparent, and podoplanin-positive fibroblasts are abundantly distributed in the encapsulating membrane. In the fibrotic phase (stage II), fibrosis is dominant, with many collagen fibers and sparsely distributed fibroblasts, and no fibrin deposition is detected, whereas podoplanin is weakly positive in fibroblast-like cells in the NFM. In the scarring phase (stage III), the NFM is characterized by loose fibrous matrices covering the peritoneal surface, occasionally forming peritoneal adhesions with scant cellular components. Mesothelial cell coverage is observed on some areas of the peritoneal surface. Both fibrin and podoplanin are no longer detected in this phase.

They suggested that NFMs were frequently observed in EPS patients and were considered necessary for the diagnosis of EPS. However, NFMs were found in a peritoneal sample sclerosis obtained from non-EPS patients, especially those with peritoneal lavage after the discontinuation of PD. NFMs have been observed in about 50\% of children undergoing PD without impairment of peritoneal function [42]. Therefore, the presence of NFMs does not predict the subsequent development of EPS. The exudative phase (stage I) is the most common in the acute phase of EPS cases, but histological detection of NFM in the fibrotic phase (stage II) and the scarring phase (stage III) can be observed in non-EPS cases.

Nakamoto [32] also presented a classification of EPS, which introduced the following 4 stages based on clinical [43-47] and imaging findings using plain abdominal radiographs, contrast imaging, ultrasound, computed tomography, and MRI [48-52]. The first stage is a pre-EPS stage (inflammation/exudation stage) without the development of a newly formed capsule, with fibrin exudation/ bleeding due to sustained inflammation/exudation reaction. The second stage is an EPS-I stage (cystic stage), in which a remarkable proliferation of enlarged fibroblasts (many podoplanin-positive cells) is observed in the capsule and where exudated fibrin accumulates on the exist-

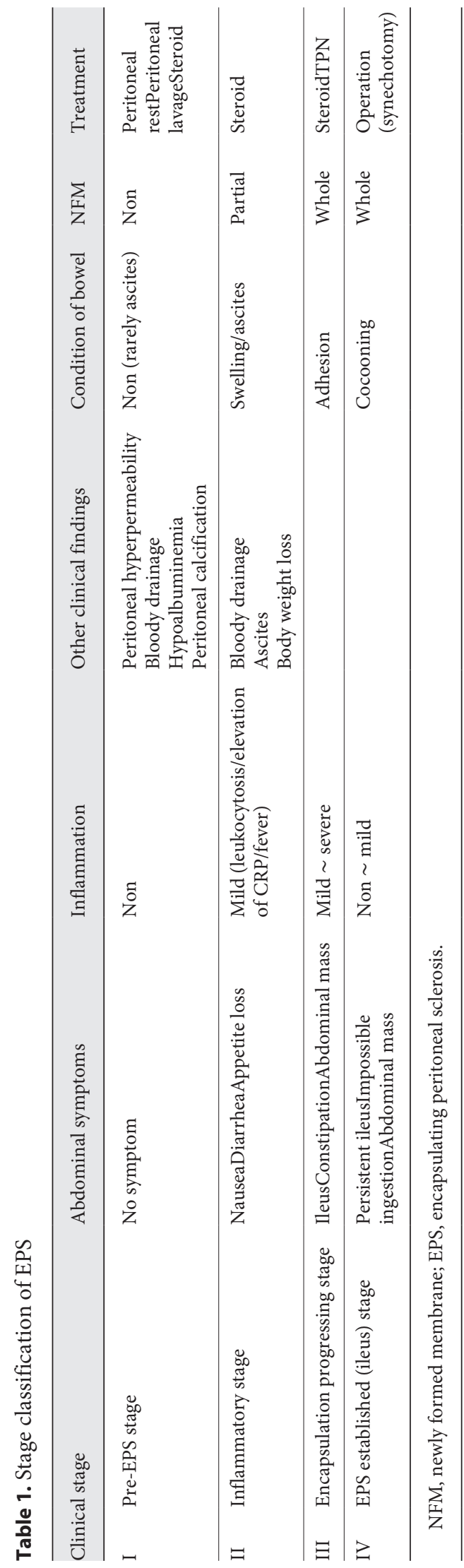

Blood Purif 2021;50:719-728 DOI: $10.1159 / 000510282$ 
ing peritoneum and fibrin capsules. The third stage is the EPS-II stage (adhesion stage), in which infiltrating macrophages and increased capillary and lymphatic vessels are observed. Once fibrin has become organized and replaced with collagenous fibers, adhesion progresses. The fourth and final stage is the EPS-III stage (scar stage), where exudation and inflammatory reactions disappear and become fibrotic scarring [31, 32].

Many case reports were evaluated for the classification/staging of EPS, and staging was performed based on the clinical and radiological findings obtained during the clinical course of the cases as well as the effects of the interventions applied, including clinical outcomes. As EPS is a rare complication in $\mathrm{PD}$, the staging is linked to therapy and is clinically useful. Nakayama et al. [53] classified 14 PD patients who developed EPS into 4 grades from clinical findings in a 4-year multicenter co-observational study in Japan (the NEXT-PD study) and examined the impact of neutral PD solution in EPS development and its prognosis.

Stage I is a pre-stage (faint abdominal symptoms and inflammation without any encapsulation and macroscopic intestinal findings). Stage II is an inflammatory stage (with abdominal symptoms, such as nausea and diarrhea, and mild or moderate inflammation). Stage III is defined as an encapsulating stage (characterized by ileus, mild to severe inflammation, whole encapsulation, and intestinal adhesion). Stage IV is defined as a chronic stage (with persistent ileus, none to mild inflammation, whole encapsulation, and shrinkage of the intestinal loop) [54].

Although this classification is interesting and based on clinical findings, additional studies are needed for further validation as this was developed using data on a small number of cases. Since it is a proposal for empirical treatment for EPS, which lacks a scientific basis, and further accumulation of cases and systematic intervention studies are desired in the future.

\section{Treatment of EPS}

The treatment of EPS is still controversial but is most often proposed to be a combination of cessation of PD as well as the use of immunosuppressive and antifibrotic agents together with nutritional support. Many medications have been tried suppressing the inflammatory component of EPS, including corticosteroids, colchicine, azathioprine, cyclosporine, mycophenolate mofetil, and mammalian target of rapamycin inhibitor. Of these, effect of corticosteroids is confined to observational studies [30, $33,55]$ and have been a common treatment for EPS. In the "2009 Peritoneal Dialysis Guidelines" of the Japanese
Society of Dialysis Treatment, a chapter on PD discontinuation guidelines has been included to avoid EPS. It recommends an evaluation of the clinical findings and the degree of peritoneal deterioration in individual PD patients [56]. In Japan, a staging of EPS based on clinical and laboratory findings has been reported, and the recommended treatment according to the stage has been presented (Table 1) [57]. We performed interventions to PD patients based on EPS staging by laparoscopic and macroscopic findings, and it was possible to prevent the development or progression of EPS, resulting in good prognosis [58]. However, there is no evidence demonstrating the protective effect of peritoneal rest or lavage on the development of EPS so far.

Although steroids have been recognized as the firstchoice drugs for EPS, this is based on empirical treatment. A recent investigation has been conducted to validate the details of the dosage and administration period. Kawanishi [59], who performed many enterolysis surgeries, suggested that the steroid treatment for EPS is effective, but it is important to perform timely surgery in patients with prolonged bowel obstruction. An appropriate evaluation of inflammation and nutritional status is essential so that the surgical intervention should not be delayed by the inappropriate administration of steroids (Table 1). In Western countries, tamoxifen has been administered for inhibiting development of peritoneal fibrosis, and the mortality rate has decreased [60], but the number of cases is still small, and further investigation is necessary.

\section{The Recent Trend of Peritoneal Membrane Pathology}

in the Era of Neutral Low GDP Solution

A neutral/low GDP dialysate with high biocompatibility has been introduced since 2000 in Japan. After the use of neutral PD solution for 10 years, the peritoneal pathology study group of the Japanese Society for Peritoneal Dialysis (JSPD) performed a pathological study on the effect of the PD solution on peritoneal tissue. Neutral PD solution preserved the vascular patency as compared to the acidic solution [61]. Kawanishi et al. [62] reported that the vascular patency was maintained by a neutral dialysate, and blood vessel density was rather increased in the neutral dialysate group. Further, the interstitial thickness did not increase, and interstitial collagen degeneration by AGE accumulation was suppressed in the neutral dialysate group [62]. These clinical studies have revealed that a neutral dialysate reduces peritoneal tissue injury through the inhibition of vasculopathy progression and interstitial collagen fiber degeneration by AGE accumulation. 


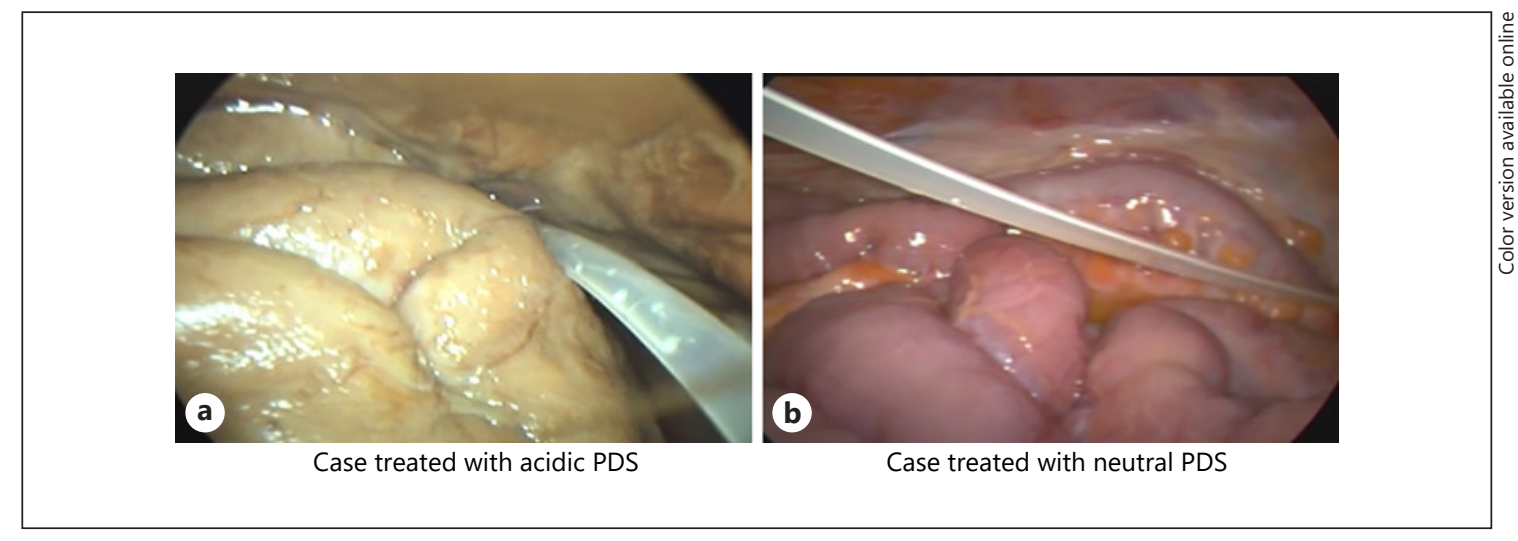

Fig. 3. Macroscopic findings in patients treated with acidic and neutral PDS. a Case treated with acidic PDS: a male patient in his 50 s with about 12 years of PD treatment. He had one event of peritonitis and underwent peritoneal lavage for 48 months. No symptoms of ileus were described, and no signs of ileus were found at direct observation. Macroscopically, the peritoneum appeared brown in color and opaque. The vascular network on the bowel surface could not be distinguished. b Case treated with neutral

\section{Laparoscopic Evaluation of the Peritoneal Membrane:} A Novel Trial for the Diagnosis of EPS

Macroscopic observations of the peritoneal cavity using a laparoscope have been performed during operations to manage conditions such as catheter infection, drainage failure, and catheter removal for PD discontinuation. Because it is possible to confirm a partial intestine envelope by NFM by laparoscopy that cannot be detected by CT, early intervention is recommended even for patients with mild clinical symptoms of EPS [58]. When an acidic solution was used as a standard dialysate, the visceral peritoneum of the intestine became severely affected by adhesions, with a yellowish leather-like opaque appearance, such that the vascular network could not be visualized (Fig. 3a). However, when using a neutral dialysate, the peritoneum becomes only slightly opaque, and the vascular network can be easily visualized. Still, some patients may present a slight degree of intestinal adhesion or hindered peristalsis (Fig. 3b). Various degrees of fibrin precipitation were observed in the peritoneal cavity in some patients. As described here, vast information can be obtained by laparoscopy compared to the microscopic observation of the biopsy specimen taken from a limited part of the abdominal wall. It is important to establish a minimally invasive observation method that enables the direct evaluation of the condition of the peritoneum and the extent of the damage by PD treatment. We examined macroscopic morphological findings such as fibrin depo-
PDS: a male patient in his 60s with 8.8 years of PD treatment without ever developing peritonitis. He had undergone peritoneal lavage for 19 months. No ileus symptoms were referred, and no signs of ileus were found at direct observation. The peritoneum appeared pink and translucid. Adhesion was observed from the intestine to the abdominal wall. The vascular network on the bowel surface could be visualized. PDS, peritoneal dialysis solution; PD, peritoneal dialysis.

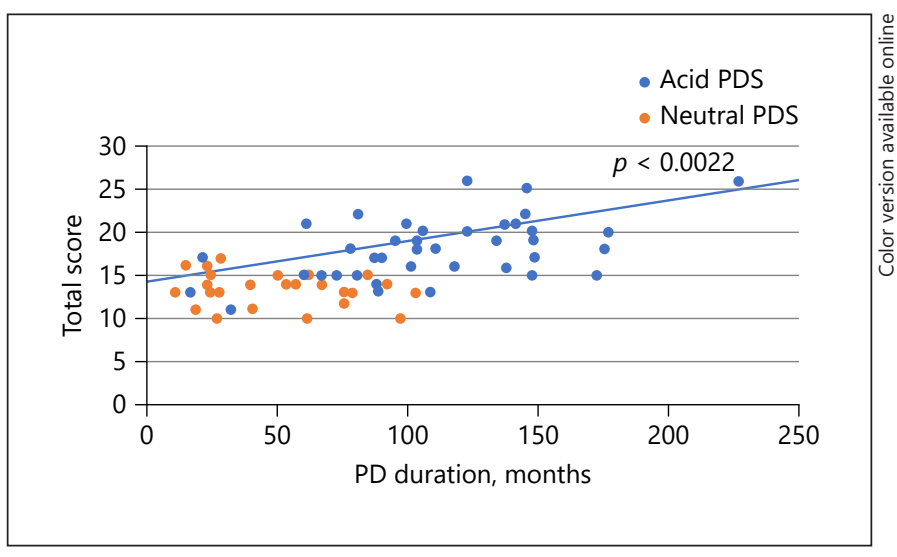

Fig. 4. Macroscopic morphological alterations in the peritoneum of patients undergoing PD. Blue circles indicate acidic dialysate group, and red circles indicate neutral dialysis group. Changes in the laparoscopic finding scores based on PD duration and the use of acidic and neutral PDS ( $p=0.0022$ in acidic PDS). PD, peritoneal dialysis; PDS, peritoneal dialysis solution.

sition, peritoneal membrane opacity, vascular structure, and presence of adhesion and calcification in both parietal and visceral peritoneums of the upper and lower peritoneal cavities and semi-quantitatively determined the scores. The total macroscopic score increased with PD duration. Peritoneal membrane opacity, fibrin deposition, and calcification were observed in the entire peritoneal cavity. The scores of fibrin deposition, opacity, and 
calcification increased with PD duration. Vascular network changes in the parietal peritoneum were more serious compared with those in the visceral peritoneum, but there was no difference in the vascular network changes between the upper and lower areas [63] (Fig. 4, $p=0.0022$ in acidic PDS). The laparoscopic finding score of patients treated with an acidic dialysate showed a positive correlation with PD period, but there was no relationship for patients treated with a neutral dialysate (Fig. 4, unpublished data) [64].

\section{Conclusions and Further Perspective}

In the 1990s, PD was recognized as the renal replacement therapy (RRT) method inferior to hemodialysis in Japan because of the occasional development of EPS. EPS is a serious complication usually seen in PD patients using long-term acidic dialysis solution, and it became a dark era for PD. A series of pathological studies of peritoneal tissue in PD patients conducted by the JSPD have begun to clarify the best approach to manage EPS. Further, treatments, including surgery, for EPS have been proposed ahead of the global clinical experiences. On the basis of the peritoneal pathological findings, clinical interventions have started to prevent peritoneal deterioration by PD treatment. Since 1993, peritoneal holiday (disruption of $\mathrm{PD}$ on hemodialysis day), enhancement of dialysis efficiency to improve the uremic condition, avoidance of the use of a high glucose dialysate to prevent ultrafiltration failure, and hybrid therapy combined with weekly hemodialysis have been introduced in Japan. In 2013, approximately $20 \%$ of PD patients in Japan were receiving hybrid therapy. Further, we confirmed that the progression of vascular lesions in peritoneal tissue was suppressed by using neutral PD solution, which has been available in Japan after the year 2000. The use of a neutral dialysate remarkably reduced the incidence of EPS in the
NEXT-PD study [53]. However, EPS still developed in PD patients using a neutral dialysate. With the use of a neutral dialysate, hybrid therapy, and icodextrin dialysate, EPS incidence is expected to further decrease. Moreover, more biocompatible dialysates are currently under study in Japan.

Because it is difficult to receive kidney transplantation, RRT by means of dialysis is a major treatment for kidney disease in Japan. As age at the beginning of dialysis and the average duration of maintenance dialysis have been increasing, the importance of PD is emphasized as an RRT that is performed on an outpatient basis. Therefore, we need to continue to improve the biocompatibility of the dialysate to prevent peritoneal deterioration and the development of EPS. With these advances, it is expected that PD will again play an effective role in RRT.

\section{Acknowledgements}

We thank our colleagues in the PD research group of the Faculty of Medicine of Juntendo University and members of the pathology study group of the Japanese Society for Peritoneal Dialysis for their support.

\section{Statement of Ethics}

The authors have no ethical conflicts to declare.

\section{Conflict of Interest Statement}

The authors have no conflicts of interest to disclose.

\section{Funding Sources}

The authors did not receive any funding.

\section{References}

1 Honda K. Structure and pathophysiology of peritoneum. Surgery. 2015;77(10):1097-105.

2 Di Paolo N, Sacchi G, Capotondo L. The peritoneum is a living membrane. Perit Dial Bull. 1987;7:S24.

3 Kastelein A, Vos LMC, de Jong KH, van Baal JOAM, Nieuwland R, van Noorden CJF, et al. Embryology, anatomy, physiology and pathophysiology of the peritoneum and the peritoneal vasculature. Semin Cell Dev Biol. 2019; 92:27-36.
4 Di Paolo N, Sacchi G. Atlas of peritoneal histology. Perit Dial Int. 2000;20(Suppl 3):S5-96.

5 Dobbie JW. Peritoneal ultrastructure and changes with continuous ambulatory peritoneal dialysis. Perit Dial Int. 1993;13(Suppl 2): S585-7.

6 Tawada M, Ito Y, Hamada C, Honda K, Mizuno M, Suzuki Y, et al. Vascular endothelial cell injury Is an important factor in the development of encapsulating peritoneal sclerosis in long-term peritoneal dialysis patients. PLoS One. 2016 Apr 27;11(4):e0154644. 
7 Williams JD, Craig KJ, Topley N, Von Ruhland C, Fallon M, Newman GR, et al. Morphologic changes in the peritoneal membrane of patients with renal disease. J Am Soc Nephrol. 2002 Feb;13(2):470-9.

8 Honda K, Hamada C, Nakayama M, Miyazaki M, Sherif AM, Harada T, et al. Impact of uremia, diabetes, and peritoneal dialysis itself on the pathogenesis of peritoneal sclerosis: a quantitative study of peritoneal membrane morphology. Clin J Am Soc Nephrol. 2008 May;3(3):720-8.

9 Pletinck A, Vanholder R, Veys N, Van Biesen W. Protecting the peritoneal membrane: factors beyond peritoneal dialysis solutions. Nat Rev Nephrol. 2012;8(9):542-50.

10 Marrón B, Martínez Ocaña JC, Salgueira M, Barril G, Lamas JM, Martín M, et al. Spanish group for CKD: analysis of patient flow into dialysis: role of education in choice of dialysis modality. Perit Dial Int. 2005 Feb;25(Suppl 3): S56-9.

11 Marrón B, Ortiz A, de Sequera P, MartínReyes G, de Arriba G, Lamas JM, et al. Impact of end-stage renal disease care in planned dialysis start and type of renal replacement therapy: a Spanish multicentre experience. Nephrol Dial Transplant. 2006 Jul;21(Suppl 2):ii51-5.

12 Rippe B. A three-pore model of peritoneal transport. Perit Dial Int. 1993;13(Suppl 2): S35-8.

13 Curry FRE, Adamson RH. Vascular permeability modulation at the cell, microvessel, or whole organ level: towards closing gaps in our knowledge. Cardiovasc Res. 2010 Jul 15;87(2): 218-29.

14 Miyawaki-Shimizu K, Predescu D, Shimizu J, Broman M, Predescu S, Malik AB. siRNA-induced caveolin-1 knockdown in mice increases lung vascular permeability via the junctional pathway. Am J Physiol Lung Cell Mol Physiol. 2006 Feb;290(2):L405-13.

15 Schubert W, Frank PG, Woodman SE, Hyogo $\mathrm{H}$, Cohen DE, Chow CW, et al. Microvascular hyperpermeability in caveolin-1 (-/-) knockout mice. Treatment with a specific nitric-oxide synthase inhibitor, L-NAME, restores normal microvascular permeability in Cav-1 null mice. J Biol Chem. 2002 Oct 18;277(42): 40091-8.

16 Margetts PJ, Bonniaud P. Basic mechanisms and clinical implications of peritoneal fibrosis. Perit Dial Int. 2003;23(6):530-41.

17 Krediet RT, Lindholm B, Rippe B. Pathophysiology of peritoneal membrane failure. Perit Dial Int. 2000;20(Suppl 4):S22-42.

18 Sherif AM, Nakayama M, Maruyama Y, Yoshida $\mathrm{H}$, Yamamoto $\mathrm{H}$, Yokoyama $\mathrm{K}$, et al. Quantitative assessment of the peritoneal vessel density and vasculopathy in CAPD patients. Nephrol Dial Transplant. 2006;21(6): 1675-81.

19 Shimaoka T, Hamada C, Kaneko K, Io H, Sekiguchi Y, Aruga S, et al. Quantitative evaluation and assessment of peritoneal morphologic changes in peritoneal dialysis patients.
Nephrol Dial Transplant. 2010 Oct;25(10): 3379-85.

20 Gavard J, Gutkind JS. VEGF controls endothelial-cell permeability by promoting the beta-arrestin-dependent endocytosis of VEcadherin. Nat Cell Biol. 2006;8(11):1223-34.

21 Flessner MF, Choi J, Vanpelt H, He Z, Credit $\mathrm{K}$, Henegar J, et al. Correlating structure with solute and water transport in a chronic model of peritoneal inflammation. Am J Physiol Renal Physiol. 2006;290(1):F232-40.

22 Zareie M, De Vriese AS, Hekking LH, ter Wee PM, Schalkwijk CG, Driesprong BA, et al. Immunopathological changes in a uraemic rat model for peritoneal dialysis. Nephrol Dial Transplant. 2005;20(7):1350-61

23 Osada S, Hamada C, Shimaoka T, Kaneko K, Horikoshi S, Tomino Y. Alterations in proteoglycan components and histopathology of the peritoneum in uraemic and peritoneal dialysis (PD) patients. Nephrol Dial Transplant. 2009 Nov;24(11):3504-12.

24 Mateijsen MA, van der Wal AC, Hendriks PM, Zweers MM, Mulder J, Struijk DG, et al. Vascular and interstitial changes in the peritoneum of CAPD patients with peritoneal sclerosis. Perit Dial Int. 1999;19(6):517-25.

25 Aroeira LS, Aguilera A, Sánchez-Tomero JA, Bajo MA, del Peso G, Jiménez-Heffernan JA, et al. Epithelial to mesenchymal transition and peritoneal membrane failure in peritoneal dialysis patients: pathologic significance and potential therapeutic interventions. J Am Soc Nephrol. 2007;18(7):2004-13.

26 Garosi G, Di Paolo N. Morphological aspects of peritoneal sclerosis. J Nephrol. 2001 NovDec;14(Suppl 4):S30-8.

27 Schneble F, Bonzel KE, Waldherr R, Bach mann S, Roth H, Schärer K. Peritoneal morphology in children treated by continuous ambulatory peritoneal dialysis. Pediatr Nephrol. 1992 Nov;6(6):542-6.

28 Gandhi VC, Humayun HM, Ing TS, Daugirdas JT, Jablokow VR, Iwatsuki S, et al. Sclerotic thickening of the peritoneal membrane in maintenance peritoneal dialysis patients. Arch Intern Med. 1980 Sep;140(9):1201-3.

29 Iwatsuki Y, Kawaguchi Y, Kubo H, Hirano H, Sakai S, Kurokawa K. Sclerosing encapsulating peritonitis (SEP) in patients on continuous ambulatory peritoneal dialysis. Nihon Toseki Igakkai Zasshi. 1998;31(4):303-11.

30 Kawanishi H, Kawaguchi Y, Fukui H, Hara S, Imada A, Kubo H, et al. Encapsulating peritoneal sclerosis in Japan: a prospective, controlled, multicenter study. Am J Kidney Dis. 2004 Oct;44(4):729-37.

31 Maruyama Y, Nakayama M. Encapsulating peritoneal sclerosis in Japan. Perit Dial Int. 2008 Jun;28(Suppl 3):S201-4.

32 Nakamoto H. Encapsulating peritoneal sclerosis: a clinician's approach to diagnosis and medical treatment. Perit Dial Int. 2005 Apr; 25(Suppl 4):S30-8.

33 Lee HY, Kim BS, Choi HY, Park HC, Kang SW, Choi KH, et al. Sclerosing encapsulating peritonitis as a complication of long-term continuous ambulatory peritoneal dialysis in Korea. Nephrology. 2003 Oct;8(8 Suppl 1): S33-9.

34 Krediet RT. The peritoneal membrane in chronic peritoneal dialysis. Kidney Int. 1999; 55(1):341-56.

35 Kawaguchi Y, Kawanishi H, Mujais S, Topley N, Oreopoulos DG. Encapsulating peritoneal sclerosis: definition, etiology, diagnosis, and treatment. International society for peritoneal dialysis ad hoc committee on ultrafiltration management in peritoneal dialysis. Perit Dial Int. 2000;20(Suppl 4):S43-55.

36 Kawanishi H, Kawaguchi Y. Prevalence and therapeutic outcome of sclerosing encapsulating peritonitis (EPS): a multicenter study in Japan(Japanese). Jin To Toseki. 2000;49(Suppl l):225-8.

37 Honda K, Oda H. Pathology of encapsulating peritoneal sclerosis. Perit Dial Int. 2005 Apr; 25(Suppl 4):S19-29.

38 Sherif AM, Yoshida H, Maruyama Y, Yamamoto H, Yokoyama K, Hosoya T, et al. Comparison between the pathology of encapsulating sclerosis and simple sclerosis of the peritoneal membrane in chronic peritoneal dialysis. Ther Apher Dial. 2008 Feb;12(1):33-41.

39 Honda K, Hamada C, Kawanishi K, Nakayama M, Miyazaki M, Ito Y. Significance of new membrane formation in peritoneal biopsies of peritoneal dialysis patients: a case-control study. Renal Replacement Ther. 2017 Aug;3: 33.

40 Honda K, Nitta K, Horita S, Tsukada M, Itabashi M, Nihei $\mathrm{H}$, et al. Histologic criteria for diagnosing encapsulating peritoneal sclerosis in continuous ambulatory peritoneal dialysis patients. Adv Perit Dial. 2003;19:169-75.

41 Schneble F, Bonzel KE, Waldherr R, Bachmann S, Roth H, Schärer K. Peritoneal morphology in children treated by continuous ambulatory peritoneal dialysis. Pediatr Nephrol. 1992 Nov;6(6):542-6.

42 Nomoto Y, Kawaguchi Y, Kubo H, Hirano H, Sakai S, Kurokawa K. Sclerosing encapsulating peritonitis in patients undergoing continuous ambulatory peritoneal dialysis: a report of the Japanese sclerosing encapsulating peritonitis study group. Am J Kidney Dis. 1996 Sep;28(3):420-7.

43 Nakamoto H, Kawaguchi Y, Suzuki H. Encapsulating peritoneal sclerosis in patients undergoing continuous ambulatory peritoneal dialysis in Japan. Adv Perit Dial. 2002;18:11923.

44 Kawanishi H, Harada Y, Noriyuki T, Kawai T, Takahashi S, Moriishi M, et al. Treatment options for encapsulating peritoneal sclerosis based on progressive stage. Adv Perit Dial. 2001;17:200-4.

45 Kawaguchi Y, Kawanishi H, Mujais S, Topley $\mathrm{N}$, Oreopoulos DG. Encapsulating peritoneal sclerosis: definition, etiology, diagnosis, and treatment. International society for peritoneal dialysis ad hoc committee on ultrafiltration management in peritoneal dialysis. Perit Dial Int. 2000 Feb;20(Suppl 4):S43-55. 
46 Novello AC, Port FK. Sclerosing encapsulating peritonitis. Int J Artif Organs. 1986 Nov; 9(6):393-6.

47 Kawanishi H. Surgical treatment for encapsulating peritoneal sclerosis. Adv Perit Dial. 2002;18:139-43.

48 Campbell S, Clarke P, Hawley C, Wigan M, Kerlin P, Butler J, et al. Sclerosing peritonitis: identification of diagnostic, clinical, and radiological features. Am J Kidney Dis. 1994 Nov;24(5):819-25.

49 Pérez-Fontán FJ, Soler R, Sanchez J, Iglesias P, Sanjurjo P, Ruiz J. Retractile mesenteritis involving the colon: barium enema, sonographic, and CT findings. AJR Am J Roentgenol. 1986 Nov; 147(5):937-40.

50 Krestin GP, Kacl G, Hoffmann R, Keusch G, Burger HR. [The imaging diagnosis of sclerosing peritonitis (SP) following continuous ambulatory peritoneal dialysis (CAPD)]. Rofo. 1992 Nov;157(5):506-11.

51 Holland P. Sclerosing encapsulating peritonitis in chronic ambulatory peritoneal dialysis. Clin Radiol. 1990 Jan;41(1):19-23.

52 Korzets A, Korzets Z, Peer G, Papo J, Stern D, Bernheim J, et al. Sclerosing peritonitis. Possible early diagnosis by computerized tomography of the abdomen. Am J Nephrol. 1988; 8(2):143-6.
53 Nakayama M, Miyazaki M, Honda K, Kasai K, Tomo T, Nakamoto H, et al. Encapsulating peritoneal sclerosis in the era of a multi-disciplinary approach based on biocompatible solutions: the NEXT-PD Study. Perit Dial Int. 2014 Nov-Dec;34(7):766-74.

54 Nakayama M, Terawaki H. Multidisciplinary clinical strategies for encapsulating peritoneal sclerosis in peritoneal dialysis: update from Japan. Int J Urol. 2014 Aug;21(8):755-61.

55 Kuriyama S, Tomonari H. Corticosteroid therapy in encapsulating peritoneal sclerosis. Nephrol Dial Transplant. 2001 Jun;16(6): 1304-5.

56 Working Group Committee for the Preparation of Guidelines for Peritoneal Dialysis, Japanese Society for Dialysis Therapy. 2009 Japanese Society for Dialysis Therapy guidelines for peritoneal dialysis. Ther Apher Dial. 2010 Dec;14(6):489-504.

57 Moinuddin Z, Summers A, Van Dellen D, Augustine T, Herrick SE. Encapsulating peritoneal sclerosis-a rare but devastating peritoneal disease. Front Physiol. 2015 Jan 5;5:470.

58 Yamahatsu A, Hamada C, Kaneko K, Io H, Nakata J, Tomino Y. Long-term outcome of encapsulating peritoneal sclerosis (EPS) patients in a single center. Clin Exp Nephrol. 2015 Oct;19(5):961-7.
59 Kawanishi H. Surgical and medical treatments of encapsulation peritoneal sclerosis. Contrib Nephrol. 2012;177:38-47.

60 Korte MR, Fieren MW, Sampimon DE, Lingsma HF, Weimar W, Betjes MGH. Tamoxifen is associated with lower mortality of encapsulating peritoneal sclerosis: results of the Dutch Multicentre EPS Study. Nephrol Dial Transplant. 2011 Feb;26(2):691-7.

61 Hamada C, Honda K, Kawanishi K, Nakamoto H, Ito Y, Sakurada T, et al. Morphological characteristics in peritoneum in patients with neutral peritoneal dialysis solution. J Artif Organs. 2015 Sep;18(3):243-50.

62 Kawanishi K, Honda K, Tsukada M, Oda H, Nitta K. Neutral solution low in glucose degradation products is associated with less peritoneal fibrosis and vascular sclerosis in patients receiving peritoneal dialysis. Perit Dial Int. 2013 May-Jun;33(3):242-51.

63 Hamada C, Nakamoto H, Suzuki Y. Morphologic characteristics of macroscopic peritoneal finding in patients with peritoneal dialysis. J Artif Organs. 2018 Mar;21(1):102-9.

64 Sasaki K, Mizuno H, Iwamoto N, Imakita M, Yasuda K, Kimura T, et al. Laparoscopy reveals a diversity of peritoneal change in patients with long-term vintage of peritoneal dialysis. Blood Purif. 2016;41(1-3):48-54. 